Published in final edited form as:

Methods Cell Biol. 2011 ; 104: 59-82. doi:10.1016/B978-0-12-374814-0.00004-5.

\title{
Retroviral-mediated insertional mutagenesis in zebrafish
}

\author{
Adam Amsterdam ${ }^{1}$, Gaurav Varshney ${ }^{2}$, and Shawn Burgess ${ }^{2}$ \\ ${ }^{1}$ Koch Institute for Integrative Cancer Research Massachusetts Institute for Technology, \\ Cambridge, MA 01239
}

${ }^{2}$ National Human Genome Research Institute, Bethesda MD 20892

\section{Introduction}

Large-scale chemical mutagenesis screens have resulted in the isolation of thousands of mutations in hundreds of genes that affect zebrafish embryonic development (Driever et al. 1996, Haffter et al. 1996). These screens have utilized an alkylating agent, ethyl nitrosourea (ENU), to induce mutations, primarily by causing base pair substitutions. Several hundred of the genes disrupted by these mutations have been isolated over the past 15 years, primarily via a candidate gene approach, and less frequently by pure positional cloning (Postlethwait and Talbot 1997), and many other chapters in this volume are devoted to describing this task. However, positional cloning remains arduous.

Insertional mutagenesis is an alternative to chemical mutagenesis in which exogenous DNA is used as the mutagen. While insertional mutagenesis is usually less efficient than ENU, insertions serve as a molecular tag to aid in the isolation of the mutated genes. Several methods might be employed to insert DNA into the zebrafish genome, including DNA microinjection (Culp et al. 1991, Stuart et al. 1988), or microinjection of DNA aided by retroviral integrases (Ivics et al., 1993) or a transposable element's transposase (Davidson et al. 2003, Kawakami et al. 2000a, Raz et al. 1998); reviews and updates on these methods can be found in other chapters of this volume. However, to date, by far the most efficient way to make a large number of insertions in the zebrafish genome is to use a pseudotyped retrovirus.

Retroviruses have an RNA genome and, upon infection of a cell, will reverse transcribe their genome to a DNA molecule, the provirus. The provirus integrates into a host cell chromosome where it remains stably and thus is inherited by all of the descendants of that cell. Replication defective retroviral vectors, unlike non-defective retroviruses, are infectious agents which can integrate into host DNA, but whose genetic material lacks the coding sequences for the proteins required to make progeny virions. Retroviral vectors are made in "split genome" packaging cells, in which the genome of the retroviral vector is expressed from one integrated set of viral sequences, while the retroviral genes required for packaging, infection, reverse-transcription, and integration are expressed from another locus. The most widely used retroviral vectors have been derived from a murine retrovirus, Moloney Murine Leukemia Virus (MoMLV), resulting in replication defective viruses that can be produced at very high titers. Initially, these retroviruses were only capable of infecting mammalian cells, but their host range can be expanded as described below.

Retroviruses have a host-range, or "tropism", which is frequently determined by their envelope protein, which recognizes and binds to some specific component, usually a protein, on the surface of the cell to be infected. Cell types that have an appropriate receptor can be infected by the retrovirus, while those that do not are refractory to infection. The host range of a virus can be changed by pseudotyping, a process in which virions acquire the genome and core proteins of one virus but the envelope protein of another. One way to enable this 
situation in split genome packaging cells is to simply substitute the gene encoding the alternative envelope protein for the usual one. While there is some specificity as to which envelope proteins can be pseudotyped with which viral genomes, one such combination that is particularly useful allows the MoMLV viral genomes and core proteins to be pseudotyped with the envelope glycoprotein (G-protein) of vesicular stomatitis virus (VSV) (Weiss et al., 1974). VSV is a rhabdovirus which is apparently pantropic; it can infect cells of species as diverse as insects and mammals (Wagner 1972). MoMLV vectors pseudotyped with VSV-G possess two qualities essential for their use in high-frequency germline transgenesis in zebrafish: the extended host range allows for the infection of fish cells, and the VSV-G pseudotyped virions are unusually stable, allowing viruses to be concentrated 1000 -fold by centrifugation (Burns et al. 1993).

When pseudotyped retroviral vectors are injected into zebrafish blastulae, many of the cells become independently infected, producing a mosaic organism in which different cells harbor proviral insertions at different chromosomal sites. When cells destined to give rise to the germ-line are infected, some proportion of the progeny of the injected fish will contain one or more insertions (Lin et al. 1994). When sufficiently high titer virus is used, one can infect a very high proportion of the germ-line of injected fish (Gaiano et al. 1996a). With very high titer virus, on average, about 25-30 independent insertions can be inherited from a single founder, though any given insertion will only be present in about three to twenty percent of the offspring (Chen et al. 2002). However, the progeny are non-mosaic for the insertions, and transmit them in a Mendelian fashion to 50\% of their progeny. Furthermore, because more than one virus can infect a single cell, some germ cells contain multiple insertions, and thus offspring can be born with as many as ten to fifteen independently segregating insertions (Amsterdam et al. 1999, Chen et al. 2002, Gaiano et al. 1996a). This remarkable transgenesis rate has made it possible to conduct an insertional mutagenesis screen which has isolated over 500 insertional mutants and allowed the rapid cloning of the mutated genes (Amsterdam et al. 2004a, Amsterdam et al. 1999, Golling et al. 2002).

\section{Mutagenesis}

In order to establish the frequency of mutagenesis with retroviral vectors in the zebrafish, we carried out a pilot screen in which we inbred over five hundred individual proviral insertions, one at a time, and screened for recessive phenotypes which could be visually scored in the first five days of embryonic development. We found six recessive embryonic lethal mutations, a frequency of about one mutation per 80-100 insertions (Allende et al. 1996, Gaiano et al. 1996b, Becker et al., 1998, Young et al. 2002). We also found one viable dominant insertional mutation (Kawakami et al. 2000b). While this rate was too inefficient to conduct a large-scale screen by breeding one insertion at a time, by utilizing the ability of founders to transmit multiple insertions to individual $F_{1}$ progeny, an average of about twelve inserts can be screened per family, allowing the recovery of about one insertional mutation per seven families screened (Amsterdam et al. 1999). This is only 7-10 fold lower than the frequency observed in analogously performed (3 generation diploid) ENU screens (Driever et al. 1996, Haffter et al. 1996, Mullins et al. 1994, Solnica-Krezel et al. 1994). The strategy to produce, select and breed the fish for such an insertional mutagenesis screen is outlined below.

\section{A. Making Founder Fish that Transmit Proviral Inserts at High Frequency to their $F_{1}$ Progeny}

Founders are produced by injecting late blastula stage (512-2000 cells) embryos. Virus must be injected into the space between the cells, and blastula stage embryos ideally accommodate the injected fluid. At this time, there are four primordial germ cells, and these cells will divide two or three times over the course of the infection (Yoon et al. 1997). Thus 
the injected embryos grow up to be founder fish (F0) with mosaic germ lines. With very good viral stocks, individual founders can contain 25-30 different insertions in their germ lines, with any given insertion present in 3-20\% of the gametes (Chen et al. 2002). Individual $\mathrm{F}_{1}$ fish can inherit up to 10 different insertions, and when founders are bred to each other $F_{1}$ fish can be found with up to 20 different insertions. $F_{1}$ fish are not mosaic, and will transmit all of their insertions in a Mendelian fashion.

Because the efficiency of the screen relies upon the generation of $F_{1}$ fish with a high number of inserts, it is essential to perform quality control assays on the viral stocks and founder injections before raising and breeding the founders. For every batch of injected embryos, several embryos are sacrificed for DNA preparation at 48 hours post-injection and subjected to quantitative Southern analysis or real-time polymerase chain reaction (PCR) to determine the average number of insertions per cell in the entire infected embryo. This number is called the embryo assay value (EAV) (Amsterdam et al., 1999). In our experience, if the average EAV is above 15 and does not vary very much amongst the individual analyzed embryos, the rest of the founders from that injection session transmit inserts at the rates mentioned above. Batches with average EAV below 15 transmit somewhat fewer inserts, and usually have greater founder-to-founder variation, and those with average EAV below 5 are quite inconsistent in transmitting multiple inserts to their progeny.

\section{B. Breeding and Screening for Mutations}

The breeding scheme for a diploid F3 insertional mutagenesis screen is outlined in Figure 1. In essence, the goal is to create families with a large number of independent insertions which can be screened simultaneously. This is achieved by selecting and breeding $F_{1}$ fish that inherit the most inserts from the mosaic founders.

Founder fish can be bred to each other or outcrossed to non-transgenic fish. For reasons that remain unclear, a majority of injected fish grow up to be males, thus it is most efficient to outcross the best male $\mathrm{F}_{0}$ fish (those from batches with the highest EAV) and inbreed the rest. $F_{1}$ families of 30 fish are raised, and at six weeks of age the fish are fin clipped for DNA preparation and analysis by quantitative Southern or real-time PCR to determine which fish harbor the most insertions. Keeping up to the three top fish per family with at least 5 inserts strikes a balance between throwing away too many inserts (if fewer fish were kept) and keeping too many "repeat inserts" (i.e. the same insert inherited by sibling $F_{1}$ fish). The repeat insert rate is quite low if only three fish are kept, as the average mosaicism (i.e. proportion of $\mathrm{F}_{1}$ inheriting a given insert) is about 8\%. In the MIT screen (Golling et al., 2002), only $3 \%$ of the recovered mutations have been caused by re-isolating such "repeat inserts."

The selected multi-insert $F_{1}$ fish are pooled together, and eventually bred to make $F_{2}$ families that harbor at least 10 different independently segregating inserts, and in which each insert is present in half of the fish. Multiple sib-crosses are then conducted between the $\mathrm{F}_{2}$ fish; since half the fish have any given insert, any insert, including those causing a mutation, should be homozygosed in one quarter of the crosses. On average, six crosses will homozygose $83 \%$ of the inserts in the family, ten crosses will screen $95 \%$ of them. Every $\mathrm{F}_{3}$ clutch from each $\mathrm{F}_{2}$ family is screened for a phenotype in one quarter of the embryos. In the MIT screen, embryos were scored for any morphological defect visible in a dissecting microscope at 1,2, and 5 days post fertilization (dpf), as well as for defects in motility and touch response. One aid to screening is that over $98 \%$ of these mutants fail to inflate their swim bladders by $5 \mathrm{dpf}$; since this is such a highly visible structure, a quick screen for clutches in which one quarter of the embryos fail to inflate their swim bladder often signals the presence of a mutation. 


\section{Cloning the Mutated Genes}

\section{A. Identifying the Mutagenic Insert}

The great advantage to using insertional mutagenesis over chemical mutagenesis is that the mutagenic insertion provides a molecular tag that can be used to identify the disrupted gene. However, because the mutagenesis screen described above utilizes multiple insertions to increase the rate of recovery of mutations, the first step after identifying a mutation is to determine which (if any) insertion appears to be responsible for the mutation. DNA is prepared from the tails of the parents of all of the crosses from the $F_{2}$ family and, using Southern analysis to distinguish the different insertions, one looks for an insertion that segregates with the phenotype (Figure 2A). A linked insert (represented by a band of a specific size) will be shared by both parents of every cross that had the phenotype and be in only one or neither of the parents of every cross which lacked the phenotype. Additionally, DNA prepared from the mutant embryos is subjected to the same analysis; while an unlinked insert would be in only three-fourths of the mutant embryos, a linked insert must be present in all of them (Figure 2B). A similar anaylsis can be done with linker-mediated PCR, although one runs the risk that not every insertion will be successfully amplified and therefore a few inserts may remain "invisible".

It is possible that no insert will appear linked to the phenotype; in the MIT screen, we found that about one quarter of the mutants recovered were not linked to a detectable insertion. Additionally, it is important to note that the identification of an insertion initially linked to the phenotype is not proof that the identified insert is tightly linked to the mutation; it is merely a way to either identify the insertion which is a candidate for causing the mutation or to conclude that the mutation is not linked to any insert if no insert meets the above criteria. This is because recombination rates in the male germ line are much lower than in the female germ line (Singer et al. 2002) thus an insert inherited from an $F_{1}$ male which is merely on the same chromosome as a non-insertional mutation will often meet the above criteria. The mutation and the insert will not have segregated in the $F_{2}$ generation, and because the mutant $\mathrm{F}_{3}$ embryos must inherit the mutant locus from both parents, even if there is recombination in the female germ line, all of the mutant embryos will receive the insert from their father. Thus additional linkage experiments that can distinguish heterozygosity from homozygosity for the insert are required, but it is not possible to perform these until genomic DNA flanking the candidate insert is cloned.

Sometimes more than one insert meets the above criteria, and thus more than one is a candidate to have caused the mutant phenotype. This can be for one of several reasons. First, if more than one insert in the family is on the same chromosome, for the reasons described above, they may fail to segregate from each other. Often this can be resolved by outcrossing a female carrier, and repeating the analysis in the next generation, either by further random sib-crosses followed by molecular analysis, or by using Southern analysis first to identify fish with one or the other insertion and then performing test crosses. Another possibility is that multiple copies of the virus have integrated in tandem, which happens about 3-4\% of the time. Usually when this happens, there is a higher-intensity provirus-sized band (if the enzyme used cuts the insert only once) in addition to the true junction fragment band. Finally, there may be too many inserts in the family to accurately distinguish all of the inserts (greater than 15-20), and this may complicate the analysis. In these cases a female carrier fish, preferably already shown to have relatively few inserts, must be outcrossed and the analysis repeated in the next generation. It is essential not to focus on a single insertion as the cause of a mutation unless it is very clear that no other insertion could also be linked to the mutant phenotype. 


\section{B. Cloning the Flanking Genomic DNA}

After identifying the candidate mutagenic insertion, inverse PCR or linker-mediated PCR can be used to clone genomic DNA flanking one or both sides of the mutagenic provirus (Figure 3a). Since all of the inserts have the same sequence, in order to clone the correct junction fragment, one must know the size expected for a given enzyme used. Often it is necessary to analyze the DNA samples by Southern analysis with several enzymes in order to identify which enzyme will be best for obtaining the desired insert (Figure 3b). After cloning and sequencing the putative junction fragment, one can design a PCR primer in the sequence that points back at the provirus, and use PCR with this and a viral primer on DNA isolated from tail fin samples of fish known to be carriers or non-carriers to confirm that the correct junction was cloned.

After cloning the genomic DNA flanking one side of the virus, it is necessary (for reasons explained below) to obtain sequence on the other side of the insertion as well; it also may be desirable to obtain additional sequence extending further from the virus on the side originally cloned. One way to do this is to use the cloned sequence as an anchor for additional inverse PCR, or linker-mediated PCR. However, as the zebrafish genome assembly becomes increasingly complete, this step is becoming increasingly dispensable; often even a small amount of sequence adjacent to the virus is sufficient to place the insertion site on a large contig of known sequence.

One of the uses of the cloned sequence is that it allows one to perform an assay to distinguish transgenic and non-transgenic chromosomes in a co-dominant fashion. Such an assay is essential in order to demonstrate that the insertion is tightly linked to the mutation, and thus most likely is its cause. One method is to use the junction fragment as a probe on a Southern blot, as the transgenic and non-transgenic chromosomes will each produce hybridizing fragments of a different size (Fig. 4a). Alternatively, PCR can be conducted with three primers, one on each side of the insert and one pointing out of the insert, such that different sized products will be amplified by insert-bearing and non-insert-bearing chromosomes (Fig. 4b). In either case, the assay is used to demonstrate that mutant embryos are invariably homozygous for the insertion, while wild type embryos never are. Every mutant analyzed is the equivalent of observing one meiotic event (only counting the female germline); every wild type analyzed is the equivalent of observing $1 / 3$ of a meiosis (only one in three recombination events between a mutation and a marker in a dihybrid cross will lead to a wild type embryo which is homozygous for the marker; thus scoring for wild types which are homozygous for a marker only detects one third of the recombination events between these loci). If any recombinants are observed between the mutation and the insertion, the insertion cannot be the cause of the mutation. We standardly analyze 50-100 meioses in this fashion; while not absolute proof that the insert is the cause of the mutation, given the size of the genome, the relative rates of spontaneous and insertional mutations, and the average number of inserts in each family, fewer than $0.5 \%$ of mutations which meet this criteria should have a cause other than the insertion. Establishing tight linkage with more observed meioses can linearly decrease the likelihood that the mutation is not caused by the insertion, but linkage alone cannot reduce this likelihood to zero.

One exception to the requirement for absolute linkage is in cases where there is incomplete penetrance of the phenotype, thus by definition the phenotype and genotype do not always match. This is evident when consistently fewer than $25 \%$ of the embryos are phenotypic. In these cases, while all of the mutant embryos still must be homozygous for the insert, some of the phenotypically wild-type embryos will also be homozygous (Amsterdam et al., 1999; Golling et al., 2002). 


\section{Gene Identification}

While the sequence of the junction fragment is useful for allowing genotypic identification of carriers and is required for the tight linkage experiments described above, its greatest utility of course is in the ability to identify the mutated gene. Given up to a few kilobases of sequence on either side of the insertion, in over $80 \%$ of the cases exon sequence can be found by BLAST (Altschul et al. 1997) based upon either nucleotide identity to a zebrafish cDNA or expressed sequence tag (EST), or amino acid homology to known or predicted proteins from other organisms when translated. RT-PCR or 3' and 5' RACE can then be used to complete the cDNA if necessary. As the zebrafish genome assembly and annotation becomes more complete, merely blasting to the genome will be sufficient to identify the gene into which the virus has inserted.

It is important that the virus actually is in, as opposed to just near, the gene identified, or it is possible to identify the wrong gene. Zebrafish genes are sometimes very near each other, with as few as half a kilobase separating them. Thus an insert could be less than one kilobase from an easily recognized or annotated gene, but actually disrupt another gene which was not found in the BLAST search. Zebrafish genes often have first exons that are entirely 5' untranslated or include the coding sequence for only a few amino acids, and such initial exons could easily be missed by a BLAST search of the genomic DNA sequence. In the case of several of the insertional mutants, the gene originally recognized in the flanking sequence either began or ended about a kilobase from the provirus; only upon more careful inspection was it found that in fact another gene began between the originally identified gene and the virus. Analysis of mRNA expression in wild-type and mutant embryos in two of these cases demonstrated that only the proximal gene's expression was affected by the insertion. On the other hand, in the case of several insertional mutants, the insert is outside the affected gene, presumably in the promoter region, and does affect the transcription of the gene. Thus the finding that the mutagenic insert lies outside of a gene does not necessarily mean that the nearest identified gene is not the gene of interest, but rather that further analysis is required.

Cloning a gene that is proximal to a mutagenic insertion is not absolute proof that the correct gene has been identified. First, it is always possible, if very unlikely, that there is a noninsertional mutation very near the insert. Second, it is possible that the expression of a neighboring gene has been affected as well. We have not observed this yet in any of the recessive mutants that we have studied, but we have found this to be the case in at least one dominant mutant (Amsterdam et al., 2009). Similarly, even when a provirus lies between two exons of a gene and disrupts its expression, it is still possible that another gene (e.g. one lying within an intron of the first gene) might be the gene responsible for the mutant. Thus to be absolutely certain that mutation of the identified gene is responsible for the phenotype, the gene identity needs to be confirmed by independent means. The finding of a second insertional allele, or non-complementation to a chemical allele with a demonstrated point mutation, would make the likelihood of a nearby non-insertional mutation exceedingly low. The ideal proof is rescue: if reintroduction of the gene in trans into mutant embryos can rescue the phenotype. Rescue is not easily accomplished in stable transgenics but can be done transiently for some mutations. Alternatively, phenocopy of the phenotype by morpholino injection (Nasevicius and Ekker, 2000) can independently verify that mutation of this gene leads to this phenotype.

\section{Phenotypic Consequence of Insertions}

The insertion of a provirus into a gene can affect that gene in a number of different ways. Unlike chemical mutagenesis, which by causing point mutations has the potential to create hypomorphic or neomorphic alleles by amino acid substitution, insertional mutagenesis 
generally works by more broadly knocking down or out gene expression, although there are exceptions. While only about one third of the mutagenic inserts actually interupt exons, which would obviously impair gene expression, nearly half land in the first intron and the rest in downstream introns (figure 5a). We have used Northern analysis and/or quantitative RT-PCR to analyze gene expression in mutants in many of these cases, and the most common effect that we have seen is a reduction or elimination of mRNA, anywhere from 5fold to undetectable levels (Golling et al. 2002). In a study of randomly selected insertions (i.e., isolated independent of causing a phenotype), most insertions in the first intron of genes were found to similarly reduce steady-state mRNA levels, while insertions in later introns were less likely to have any effect (Wang et al. 2007). Thus while some insertional mutants are nulls, some are hypomorphs, as expression has not been completely abrogated.

Additionally, some insertional mutants can affect the nature of the message (and the protein it produces) rather than merely the level of expression. First, some mutations cause exon skips instead of down-regulation of expression. For example, we have three insertional alleles of thevHNF-1 gene, two in the first or second exon, which appear to be nulls, and one in the fifth intron, which leads to two different splice variants, skipping either the fourth or third and fourth exons (Sun and Hopkins 2001). This allele is predicted to make a truncated protein, and in fact has a less severe phenotype. Second, the virus used to make most of the insertional mutations contains a splice-in, splice-out, frameshift-producing gene trap cassette (Chen et al. 2002). When the virus inserts in an intron in the correct orientation, it is possible for the preceding splice donor to splice to this exon in the provirus, and splice out to the next endogenous exon, thus creating a frameshift and presumably a truncated protein (Fig. 5b). If this happens, analysis of mRNA by RT-PCR will show the presence of an increased-sized band, indicating the inclusion of the trapped exon. This is likely to lead to a truncated protein, and thus could act as a hypomorph or neomorph. While we have not found this to be a common mechanism of mutation, it has occurred in some of the mutants.

\section{Retroviruses mediated insertional mutagenesis as a reverse genetic tool}

\section{A. Demonstrating the utility of retroviral mutagenesis to reverse genetics approaches}

As described earlier, retroviruses have been used in forward genetic screens to isolate mutations affecting embryonic development (Amsterdam et al. 1999). Forward genetics is a process where mutational studies are done to determine the genetic basis of associated phenotypes. Forward genetic screens are effective in identifying mutations with visible phenotypes, but are limited by issues of gene redundancy or the ability to detect phenotypes blindly. With the recent advancement in sequencing technologies and availability of highquality whole genomes, researchers have access to rich gene information in many model organisms and it becomes possible to systematically screen by first identifying mutations in genes, and then testing homozygous mutant animals for phenotypes, i.e. "reverse" genetics.

The genes can be disrupted either in a targeted manner (homologous recombination or RNAi mediated knockdown) or by using non-targeted approaches (insertional mutagenesis or chemical induced mutagenesis for example).

The most widely used reverse genetic approach in zebrafish is Targeted Induced Local Lesion IN Genomes (TILLING), which has been used to identify a desired mutation from a pool of ENU induced mutagenize fish either through CEL1 nuclease assay or DNA sequencing (Stemple 2004), Wienholds et al. 2003). While effective, this approach is expensive and labor-intensive.

It has been shown that mutagenesis using MoMLV as an insertional mutagen followed by integration mapping and recovery is possible, and thus could be an alternative reverse 
genetics approach (Wang et al. 2007). A public effort is underway in the Burgess lab in collaboration with Dr. Shou Lin's lab at UCLA to systematically disrupt every proteincoding gene in zebrafish genome using pseudotyped MoMLV. I $n$ this approach we infect zebrafish embryos with MoMLV, and proviral integrations in the $F_{1}$ generation of infected fish are mapped by PCR amplification and sequencing (Figure 6). Using this strategy it is possible to identify a mutagenic event for every 30 sequencing reactions done on the $F_{1}$ fish, thus showing up to 30 -fold increase in efficiency compared to TILLING (Wang et al. 2007). The gene disruption pipeline begins with the production of high-titer pseudotyped MoMLV retroviruses using an improved method previously described in (Jao and Burgess 2009), which is then injected into blastula stage embryos (as described earlier) to get founder fish. We raise the founders; outcross them with wild-type fish to obtain the $F_{1}$ generation. We raise only the male $F_{1}$ fish, sacrifice them to cryopreserve their testes, and collect corresponding tail cuts to isolate genomic DNA (gDNA) for the integration mapping. In order to map retroviral integrations, gDNA is subjected to restriction digestion and then linkers are ligated to the digested fragments. We use a linker-specific and proviral-LTRspecific primer to amplify proviral-gDNA-LTR fragments. The amplified PCR products are sub-cloned and to date have been sequenced by Sanger sequencing. The sequences are mapped back to zebrafish whole genome assembly to get the integration information. Our goal is to generate a genome-wide knockout library and mutagenize to saturation in next 5 years.

Our lab originally investigated the distribution profile of proviral integration sites in the zebrafish genome by sequencing integrations from $900 \mathrm{~F}_{1}$ fish. We obtained 933 unique sequences, out of these, 599 integration sites were mapped to zebrafish genome. Sixty-five percent of mapped integrations either landed in annotated Ensembl genes, or within $3 \mathrm{~kb}$ of genes, with a strong preference for the first-intron of genes (Wang et al. 2007). The preference of MoMLV integration for the first-intron is consistent with the results in mouse (Mooslehner et al. 1990, Scherdin et al. 1990) and human tissue culture cells (Wu et al. 2003). We showed that not only integrations in the exons are mutagenic but also integrations landing in the first intron of genes are also highly mutagenic (Golling et al. 2002, Wang et al. 2007). The gene expression level in $80 \%$ of the first-intron hits reduced mRNA level to $<10 \%$ of wild-type level. Our prediction is one in five retroviral integrations will result in a gene disruption that reduces the gene expression level to $10 \%$ or less of the wild-type level.

\section{B. Adapting retroviral mapping strategies to $2^{\text {nd }}$ generation sequencing technologies}

The advantage of retroviral mutagenesis over ENU is that mutated genes are molecularly tagged by proviral sequences. By identifying genomic sequences flanking proviral integrations, these integrations can be identified in a relatively short time compared to ENU mediated mutagenesis (Gaiano et al. 1996b, Golling et al. 2002). Our lab has established another method of identifying these integrations that adapts well to high-throughput approaches. The identification of the MoMLV integration by LM-PCR starts by fragmenting gDNA using a frequent cutter restriction enzyme, followed by ligating linkers and linker mediated PCR, shotgun cloning and Sanger sequencing. However, this method has limitations, we identify fewer integrations than is predicted based on qPCR data.

This limited recovery is not an issue for phenotype-based screens, as only specific integrations that are linked with the phenotype need to be recovered. But in order to implement the reverse genetic strategy to generate a genome-wide knockout library, it is imperative to recover as many integration as possible. In addition, the published method is also labor intensive and expensive as it relies on a large number of sub-cloning, and sequencing reactions that require significant resources and manpower. Because of the labor and expense, gDNA is digested with a single restriction frequently cutting enzyme, MseI. As a result, many integrations will be either too close or too far away from the nearest MseI 
site, resulting in unmappable integrations. This limitation can be overcome by the use of multiple restriction enzymes to get appropriately size fragments but with traditional Sanger sequencing, the costs become prohibitive. Another limitation that contributes to limited recovery is overrepresentation of some clones in LM-PCR due to PCR-biases, thus some clones may be over-represented in the cloning mix and others under-represented. When performing a relatively small number of sequences per fish $(<10)$, your sample size isn't large enough to ensure good representation of all integrations in a given fish. To solve this problem, deeper sequence sampling of the PCR product is required so that underrepresented clone will also be recovered.

To overcome these limitations and improve the mapping process, we have modified the strategy by adapting the approach to second generation sequencing technologies. The modified method uses three pairs of enzymes (Mse1 and PstI), (Bfa1 and BanII), and (Csp6I and BanII). PstI or BanII is used to suppress the amplification of an internal band that would occur from the 5' LTR (which has identical sequence to the 3' LTR). Mse1, Csp6I and Bfa1 recognize the 4-base TTAA, CTAG and GTAC sites in the genome respectively, all three enzymes generating the same 5'-TA overhang in the digested DNA. The linkers, consisting of a short and long strand, are ligated onto the overhanging ends of the gDNA. When these strands are annealed, the short strand contains non-complimentary sequences that will form a hairpin. This design prevents unwanted linker-to-linker amplification. The long strand of the linker does not contain a binding sites for the linker PCR primer; instead it contains an exact match to the linker primer sequence and will only work after its complimentary sequence is synthesized. As a result, only gDNA containing LTR-gDNA-linker sequences will be amplified. In order to make the mapping pipeline cost-effective and high-throughput, we wish to replace the multi-stepped shotgun cloning and sequencing process with second generation sequencing technology (Figure 7). There are several commercial next-generation sequencing platforms available each with some advantages and disadvantages. We utilize the Illumina Genome Analyzer (GAxII) platform because of high sequence read numbers, relatively low costs and simplified template preparation. By using the GAxII, we overcome the problem of limited sampling as hundreds of samples are pooled together in a single lane of sequencing and each sample (i.e. fish) will still have tens of thousands of sequence reads each. However, pooled samples pose a challenge as how to assign the mapped integrations to their respective fish. We developed a 6-base molecular barcode strategy and embedded 960 different barcode sequences into linkers, thus the digested DNA from each $\mathrm{F}_{1}$ fish can be coded individually. This barcoding strategy allows us to minimize the sample handling and reagent consumption in LM-PCR reactions as barcoded samples can be pooled together, bringing mapping costs down. We added Illumina adapter sequences to the LTR and Linker specific primers and these adapter sequences are used to bind the amplicons to the GAxII flowcells. The LM-PCR products are sequenced from both sides using "paired-end" sequencing. We obtain 24 bp of LTR sequence and 76 bp of genomic sequence on one side, and $24 \mathrm{bp}$ of linker, the $6 \mathrm{bp}$ barcode, and $70 \mathrm{bp}$ of genomic sequence from the opposite side. It is therefore possible to get up to $150 \mathrm{bp}$ of genomic sequence for mapping the retroviral integration, although the majority of the reads in the sequencing are shorter at 60 $70 \mathrm{bp}$ long. The resulting sequence reads are filtered leaving only perfect LTR and linker sequences. The remaining reads are aligned using the short-read aligner program Bowtie (Langmead et al. 2009) and mapped to the latest assembly of the zebrafish genome.

We have compared traditional Sanger sequencing method to the barcoded Illumina method. We recovered $\sim 57 \%$ more mappable integrations using the modified method. The majority of this increase in recovery can be explained by the use of the three sets of restriction enzymes and the exhaustive sequence sampling. To date, we have mapped more than 6000 integrations from 2000 fish in a very high-throughput and cost-effective manner. 


\section{Future Directions}

The current insertional mutant collection from the Hopkins efforts includes over 500 mutants, with mutations in nearly 400 genes; so far we have identified 340 of these genes. We believe that this represents approximately $25 \%$ of the genes which can be mutated to a visible (and usually lethal) phenotype in the fish (Amsterdam et al. 2004a). While the characterization of the phenotypes is somewhat rudimentary at present, numerous "shelf screens" of this collection have been conducted, including staining with various antibodies, in situ hybridization markers, and other reagents that illuminate the patterning and development of specific tissues, as well as the assessment of various physiological functions. Thus a substantial portion of the genes required for the proper formation of all of these structures will be identified.

The existing collection can also be used to monitor the long-term effects of heterozygosity of these genes in adults, as mutations that have recessive embryonic phenotypes might predispose adults to disease in the heterozygous state. The genes mutated in many of the mutants are known to be autosomal dominant disease genes in humans. For example, mutations in the zebrafish vHNF1 gene affect both kidney and pancreas development (Sun and Hopkins 2001). vHNF1 mutations in the heterozygous state, while not yet investigated in fish, can lead to either kidney disease or diabetes in humans (Horikawa et al. 1997, Nishigori et al. 1998). Similarly, the jellyfish mutation, which results in defective cartilage differentiation and morphogenesis, is caused by disruption of the sox9a gene (Yan et al. 2002), while heterozygosity for sox 9 mutations in humans leads to campomelic dysplasia (Foster et al. 1994). Thus it might not be surprising if heterozygotes of insertional mutants were predisposed to a variety of diseases which might be screenable. One example which has borne this out is the identification of insertional mutants with ageing phenotypes; heterozygotes for mutations in either the spinster homolog or telomerase-binding factor 2 have a shortened lifespan as well as some other markers of ageing such as lipofuscin accumulation and premature retinal degeneration (Kishi et al. 2008).

Similarly, many genes whose mutation in the heterozygous state is known to predispose mammals to cancer (tumor suppressor genes) cause prenatal death in mice in the homozygous state (Jacks 1996). An insertional mutation of the NF2a gene, an ortholog of a known human tumor suppressor gene (Ruttledge et al. 1994, Trofatter et al. 1993), is embryonic lethal in zebrafish. Heterozygosity for this NF2a mutation in zebrafish predisposes them to development of tumors of the nervous system (Amsterdam et al. 2004b), as it does in mammals. Amongst a large collection of recessive embryonic lethal mutations one might expect to find other mutations in which heterozygotes are more prone to develop cancer, and in fact, a number of the insertional mutations have increased rates of tumirogenesis as heterozygous adults (Amsterdam et al. 2004b, Lai et al. 2009).

While the screen we have conducted has netted hundreds of embryonic lethal mutations, it is also possible that the retroviral technology could be used in new screens targeted for specific phenotypes in more efficient ways. The screen described above was quite labor intensive and required a lot of tanks for the several generations of breeding that were involved. There are several ways in which the mosaicism of the founders' germline might be used for more efficient screening, although these approaches would require the ability to recognize the mutants when present at far less than the usual $25 \%$ of the clutch. While it might be difficult to conduct a general morphological screen in such a way, this might work to screen for individual structures or patterns, e.g. by antibody staining or in situ hybridization. Alternatively, such screens might be conducted in strains that allow the easy detection of mutations for certain processes, such as GFP transgenic lines that illuminate structures of interest. 
One method would be to sequentially backcross individual $F_{1}$ fish to their founder parent. Because the average germline clone size for the insertions is small, the phenotype would have to be scored where only $3-10 \%$ of the embryos are mutant. Nonetheless, this would likely allow for the screening of more insertions per $\mathrm{F}_{0}$ fish than the three-generation screen that we conducted, and mutations would be identified one generation earlier. Phenotypes of interest could then be recovered by outcrossing the carrier $F_{1}$ fish and reidentifying the mutation in the $\mathrm{F}_{2}$ generation, where the mutagenic insertion could be identified and its adjacent DNA cloned.

Alternatively, one could conduct gynogenetic screens utilizing either haploids or early pressure diploids (Beattie et al. 1999, Walker 1999). One possibility would be to screen the progeny of $\mathrm{F}_{0}$ fish, thus screening dozens of insertions per clutch, without any of the generations of breeding or selection of high insert number fish. Again, because of the germline mosaicism, only a small percentage of the embryos will inherit any given insertion, so the screen would have to be sensitive enough to detect only one or a few mutants per clutch, without too high a false positive rate. In a pilot screen in which the haploid progeny of about 300 mosaic $\mathrm{F}_{0}$ fish were screened for both brain pattern formation by in situ hybridization at tailbud stage and brain morphology at $32 \mathrm{hpf}$, six insertional mutants were recovered (Wiellette et al. 2004). Alternatively, if one required a higher proportion of mutant embryos to be sure of the phenotype, one could screen the progeny of multi-insert $F_{1}$ fish, selected as in the three-generation screen. While this would add the work and resources to raise all $F_{1}$ families and select the high insert number $F_{1}$ fish, one could still screen around ten inserts per clutch and it would still be one fewer generation to breed and many fewer clutches to screen than the diploid $\mathrm{F}_{3}$ screen described above. Either of these methods has the potential to approach saturation for a given phenotype.

There is also room for newer vectors or other methods of transgenesis to improve insertional mutagenesis, many of which are described in other chapters in this volume. Vectors employing a gene trap or enhancer trap with a visible marker such as GFP could preselect for insertions in genes with expression patterns of interest (Ellingsen et al. 2005, Kawakami et al. 2004). Such insertions could be selected in $F_{1}$ fish after passage through the germline, or gene trap events could be selected in vitro, as is often done in ES cells in mice, and then cloned by nuclear transfer (Lee et al. 2002). Alternatively, it has been suggested that vectors including transcriptional regulatory elements such as the tetracycline-responsive promoter or the binary Gal4/UAS system could create inducible gain-of-function mutations (Chen et al. 2002). One such screen utilized a virus containing multiple Gal4-responsive UAS sequences. Upon crossing fish harboring insertions of this virus to a transgenic line expressing Gal4-VP16 in a subset of neurons, genes at the insertion site of the virus were overexpressed in those neurons (Maddison et al 2009). Thus, in a single generation screen, genes could be identified whose overexpression in a particular cell type caused a phenotype - a method which could be repeated with many different Gal4-VP16 expressing lines. And retroviruses need not be the only possible tool. Several transposons have been shown to be able to integrate into the zebrafish genome (Davidson et al. 2003, Kawakami et al. 2000b, Raz et al. 1998); if they could be mobilized in a controlled fashion similar to $P$ elements in flies (Cooley et al. 1988, Spradling et al. 1999), they could prove to be a very effective mutagenesis tool. Transposons have also been shown to be effective in delivering gene traps and enhancer traps, although the frequency with which these vectors mutate the gene they trap is still unclear (Balciunas et al. 2004, Kawakami et al. 2004, Parinov et al. 2004, Sivasubbu et al. 2006). Recent unpublished results suggest these vectors are becoming very promising as mutagens.

Finally, insertional technologies, be they retroviral or transposon based, could also be used for reverse genetics by generating a library of insertions, as has become popular in other 
model organisms. Thousands of P-element fly lines have been cataloged with their junction sequences and thus chromosomal location now that the genome is complete (FlyBase Consortium, 2002). Similarly, a consortium of several labs are producing thousands of murine ES cell clones and identifying the trapped genes by inverse PCR or RACE (Wiles et al., 2000; Stanford et al., 2001). Also in mice, hundreds of Sleeping Beauty insertions in live mouse lines are being mapped (Roberg-Perez et al. 2003). In C elegans, hundreds of thousands of Tc1 insertion lines have been isolated and their genomic DNA has been arrayed to provide a PCR-screenable panel for insertions in any gene of interest (Zwaal et al. 1993). As described above, an analogous approach has been taken in zebrafish. A similar strategy had been undertaken by Znomics Inc which mapped and cryopreserved 200,000 insertions from $\mathrm{F}_{0}$ fish which include insertions in an exon or the first intron of over 7,000 genes (http://zenemark.znomics.com/index.html). A publically funded effort to create a library of mapped $\mathrm{F}_{1} \mathrm{~s}$ is also underway (Wang et al. 2007 and section V). Screening such libraries provides an alternative to screening chemically mutagenized sperm libraries (Wienholds et al;; 2002, Stemple, 2004).

One potentially powerful aspect of such a large mutant collection (or collections) would be the ability to systematically screen every gene in the zebrafish genome for phenotypes. By knowing which gene is mutated before screening for the phenotype, it would allow researchers to not only screen for relatively simple visual phenotypes, but also for more "targeted" phenotype assessment based on the nature of the mutated gene. For example, genes known to be expressed in the sensory epithelium of the ear can be tested for hearing defects in adults if no obvious phenotype is seen in the developing embryo. The testing of double mutations in the case of gene duplication would be possible as well. An additional advantage of this type of screening is that you would not be limited to testing haploinsufficiency phenotypes in adults, it would also be possible to systematically track homozygous mutations for adult phenotypes.

\section{Acknowledgments}

We thank past and present members of the Hopkins, Lin, and Burgess laboratories for their contribution to the insertiontional mutagenesis screen and mutant library efforts. This research was supported by the Intramural Research Program of the National Human Genome Research Institute, National Institutes of Health (SB), or Amgen Inc. and the NIH extramural program (AA).

\section{References}

Allende ML, Amsterdam A, Becker T, Kawakami K, Gaiano N, Hopkins N. Insertional mutagenesis in zebrafish identifies two novel genes, pescadillo and dead eye, essential for embryonic development. Genes Dev. 1996; 10:3141-3155. [PubMed: 8985183]

Altschul SF, Madden TL, Schaffer AA, Zhang J, Zhang Z, Miller W, Lipman DJ. Gapped BLAST and PSI-BLAST: a new generation of protein database search programs. Nucleic Acids Res. 1997; 25:3389-3402. [PubMed: 9254694]

Amsterdam A, Nissen RM, Sun Z, Swindell EC, Farrington S, Hopkins N. Identification of 315 genes essential for early zebrafish development. Proc Natl Acad Sci U S A. 2004a; 101:12792-12797. [PubMed: 15256591]

Amsterdam A, Sadler KC, Lai K, Farrington S, Bronson RT, Lees JA, Hopkins N. Many ribosomal protein genes are cancer genes in zebrafish. PLoS Biol. 2004b; 2:E139. [PubMed: 15138505]

Amsterdam A, Burgess S, Golling G, Chen W, Sun Z, Townsend K, Farrington S, Haldi M, Hopkins N. A large-scale insertional mutagenesis screen in zebrafish. Genes Dev. 1999; 13:2713-2724. [PubMed: 10541557]

Amsterdam A, Lai K, Komisarczuk AZ, Becker TS, Bronson RT, Hopkins N, Lees JA. Zebrafish Hagoromo mutants upregulate fgf8 post-embryonically and develop neuroblastoma. Mol Cancer Res. 2009; 7:841-850. [PubMed: 19531571] 
Balciunas D, Davidson AE, Sivasubbu S, Hermanson SB, Welle Z, Ekker SC. Enhancer trapping in zebrafish using the Sleeping Beauty transposon. BMC Genomics. 2004; 5:62. [PubMed: 15347431]

Beattie CE, Raible DW, Henion PD, Eisen JS. Early pressure screens. Methods Cell Biol. 1999; 60:71-86. [PubMed: 9891331]

Becker TS, Burgess SM, Amsterdam AH, Allende ML, Hopkins N. not really finished is crucial for development of the zebrafish outer retina and encodes a transcription factor highly homologous to human Nuclear Respiratory Factor-1 and avian Initiation Binding Repressor. Development. 1998; 125:4369-4378. [PubMed: 9778497]

Burns JC, Friedmann T, Driever W, Burrascano M, Yee JK. Vesicular stomatitis virus G glycoprotein pseudotyped retroviral vectors: concentration to very high titer and efficient gene transfer into mammalian and nonmammalian cells. Proc Natl Acad Sci U S A. 1993; 90:8033-8037. [PubMed: 8396259]

Chen W, Burgess S, Golling G, Amsterdam A, Hopkins N. High-throughput selection of retrovirus producer cell lines leads to markedly improved efficiency of germ line-transmissible insertions in zebra fish. J Virol. 2002; 76:2192-2198. [PubMed: 11836396]

Cooley L, Kelley R, Spradling A. Insertional mutagenesis of the Drosophila genome with single P elements. Science. 1988; 239:1121-1128. [PubMed: 2830671]

Culp P, Nusslein-Volhard C, Hopkins N. High-frequency germ-line transmission of plasmid DNA sequences injected into fertilized zebrafish eggs. Proc Natl Acad Sci U S A. 1991; 88:7953-7957. [PubMed: 1910170]

Davidson AE, Balciunas D, Mohn D, Shaffer J, Hermanson S, Sivasubbu S, Cliff MP, Hackett PB, Ekker SC. Efficient gene delivery and gene expression in zebrafish using the Sleeping Beauty transposon. Dev Biol. 2003; 263:191-202. [PubMed: 14597195]

Driever W, et al. A genetic screen for mutations affecting embryogenesis in zebrafish. Development. 1996; 123:37-46. [PubMed: 9007227]

Ellingsen S, Laplante MA, Konig M, Kikuta H, Furmanek T, Hoivik EA, Becker TS. Large-scale enhancer detection in the zebrafish genome. Development. 2005; 132:3799-3811. [PubMed: 16049110]

Foster JW, et al. Campomelic dysplasia and autosomal sex reversal caused by mutations in an SRYrelated gene. Nature. 1994; 372:525-530. [PubMed: 7990924]

Gaiano N, Allende M, Amsterdam A, Kawakami K, Hopkins N. Highly efficient germ-line transmission of proviral insertions in zebrafish. Proc Natl Acad Sci U S A. 1996a; 93:7777-7782. [PubMed: 8755552]

Gaiano N, Amsterdam A, Kawakami K, Allende M, Becker T, Hopkins N. Insertional mutagenesis and rapid cloning of essential genes in zebrafish. Nature. 1996b; 383:829-832. [PubMed: 8893009]

Golling G, et al. Insertional mutagenesis in zebrafish rapidly identifies genes essential for early vertebrate development. Nat Genet. 2002; 31:135-140. [PubMed: 12006978]

Haffter $\mathrm{P}$, et al. The identification of genes with unique and essential functions in the development of the zebrafish, Danio rerio. Development. 1996; 123:1-36. [PubMed: 9007226]

Horikawa Y, et al. Mutation in hepatocyte nuclear factor-1 beta gene (TCF2) associated with MODY. Nat Genet. 1997; 17:384-385. [PubMed: 9398836]

Jacks T. Tumor suppressor gene mutations in mice. Annu Rev Genet. 1996; 30:603-636. [PubMed: 8982467]

Jao LE, Burgess SM. Production of pseudotyped retrovirus and the generation of proviral transgenic zebrafish. Methods Mol Biol. 2009; 546:13-30. [PubMed: 19378095]

Kawakami K, Shima A, Kawakami N. Identification of a functional transposase of the Tol 2 element, an Ac-like element from the Japanese medaka fish, and its transposition in the zebrafish germ lineage. Proc Natl Acad Sci U S A. 2000a; 97:11403-11408. [PubMed: 11027340]

Kawakami K, Takeda H, Kawakami N, Kobayashi M, Matsuda N, Mishina M. A transposon-mediated gene trap approach identifies developmentally regulated genes in zebrafish. Dev Cell. 2004; 7:133-144. [PubMed: 15239961]

Kawakami K, Amsterdam A, Shimoda N, Becker T, Mugg J, Shima A, Hopkins N. Proviral insertions in the zebrafish hagoromo gene, encoding an F-box/WD40-repeat protein, cause stripe pattern anomalies. Curr Biol. 2000b; 10:463-466. [PubMed: 10801422] 
Kishi S, et al. The identification of zebrafish mutants showing alterations in senescence-associated biomarkers. PLoS Genet. 2008; 4:e1000152. [PubMed: 18704191]

Lai K, Amsterdam A, Farrington S, Bronson RT, Hopkins N, Lees JA. Many ribosomal protein mutations are associated with growth impairment and tumor predisposition in zebrafish. Dev Dyn. 2009; 238:76-85. [PubMed: 19097187]

Langmead B, Trapnell C, Pop M, Salzberg SL. Ultrafast and memory-efficient alignment of short DNA sequences to the human genome. Genome Biol. 2009; 10:R25. [PubMed: 19261174]

Lee KY, Huang H, Ju B, Yang Z, Lin S. Cloned zebrafish by nuclear transfer from long-term-cultured cells. Nat Biotechnol. 2002; 20:795-799. [PubMed: 12134167]

Lin S, Gaiano N, Culp P, Burns JC, Friedmann T, Yee JK, Hopkins N. Integration and germ-line transmission of a pseudotyped retroviral vector in zebrafish. Science. 1994; 265:666-669. [PubMed: 8036514]

Maddison LA, Lu J, Victoroff T, Scott E, Baier H, Chen W. A gain-of-function screen in zebrafish identifies a guanylate cyclase with a role in neuronal degeneration. Mol Genet Genomics. 2009; 281:551-563. [PubMed: 19221799]

Mooslehner K, Karls U, Harbers K. Retroviral integration sites in transgenic Mov mice frequently map in the vicinity of transcribed DNA regions. J Virol. 1990; 64:3056-3058. [PubMed: 2335826]

Mullins MC, Hammerschmidt M, Haffter P, Nusslein-Volhard C. Large-scale mutagenesis in the zebrafish: in search of genes controlling development in a vertebrate. Curr Biol. 1994; 4:189-202. [PubMed: 7922324]

Nishigori H, Yamada S, Kohama T, Tomura H, Sho K, Horikawa Y, Bell GI, Takeuchi T, Takeda J. Frameshift mutation, A263fsinsGG, in the hepatocyte nuclear factor-1beta gene associated with diabetes and renal dysfunction. Diabetes. 1998; 47:1354-1355. [PubMed: 9703339]

Parinov S, Kondrichin I, Korzh V, Emelyanov A. Tol2 transposon-mediated enhancer trap to identify developmentally regulated zebrafish genes in vivo. Dev Dyn. 2004; 231:449-459. [PubMed: 15366023]

Postlethwait JH, Talbot WS. Zebrafish genomics: from mutants to genes. Trends Genet. 1997; 13:183190. [PubMed: 9154001]

Raz E, van Luenen HG, Schaerringer B, Plasterk RH, Driever W. Transposition of the nematode Caenorhabditis elegans Tc3 element in the zebrafish Danio rerio. Curr Biol. 1998; 8:82-88. [PubMed: 9427643]

Roberg-Perez K, Carlson CM, Largaespada DA. MTID: a database of Sleeping Beauty transposon insertions in mice. Nucleic Acids Res. 2003; 31:78-81. [PubMed: 12519952]

Ruttledge MH, et al. Evidence for the complete inactivation of the NF2 gene in the majority of sporadic meningiomas. Nat Genet. 1994; 6:180-184. [PubMed: 8162072]

Scherdin U, Rhodes K, Breindl M. Transcriptionally active genome regions are preferred targets for retrovirus integration. J Virol. 1990; 64:907-912. [PubMed: 2296087]

Singer A, Perlman H, Yan Y, Walker C, Corley-Smith G, Brandhorst B, Postlethwait J. Sex-specific recombination rates in zebrafish (Danio rerio). Genetics. 2002; 160:649-657. [PubMed: $11861568]$

Sivasubbu S, Balciunas D, Davidson AE, Pickart MA, Hermanson SB, Wangensteen KJ, Wolbrink DC, Ekker SC. Gene-breaking transposon mutagenesis reveals an essential role for histone H2afza in zebrafish larval development. Mech Dev. 2006; 123:513-529. [PubMed: 16859902]

Solnica-Krezel L, Schier AF, Driever W. Efficient recovery of ENU-induced mutations from the zebrafish germline. Genetics. 1994; 136:1401-1420. [PubMed: 8013916]

Spradling AC, Stern D, Beaton A, Rhem EJ, Laverty T, Mozden N, Misra S, Rubin GM. The Berkeley Drosophila Genome Project gene disruption project: Single P-element insertions mutating $25 \%$ of vital Drosophila genes. Genetics. 1999; 153:135-177. [PubMed: 10471706]

Stemple DL. TILLING--a high-throughput harvest for functional genomics. Nat Rev Genet. 2004; 5:145-150. [PubMed: 14726927]

Stuart GW, McMurray JV, Westerfield M. Replication, integration and stable germ-line transmission of foreign sequences injected into early zebrafish embryos. Development. 1988; 103:403-412. [PubMed: 2852096] 
Sun Z, Hopkins N. vhnf1, the MODY5 and familial GCKD-associated gene, regulates regional specification of the zebrafish gut pronephros, and hindbrain. Genes Dev. 2001; 15:3217-3229. [PubMed: 11731484]

Trofatter JA, et al. A novel moesin-, ezrin-, radixin-like gene is a candidate for the neurofibromatosis 2 tumor suppressor. Cell. 1993; 75:826. [PubMed: 8242753]

Walker C. Haploid screens and gamma-ray mutagenesis. Methods Cell Biol. 1999; 60:43-70. [PubMed: 9891330]

Wang D, et al. Efficient genome-wide mutagenesis of zebrafish genes by retroviral insertions. Proc Natl Acad Sci U S A. 2007; 104:12428-12433. [PubMed: 17640903]

Wiellette E, Grinblat Y, Austen M, Hirsinger E, Amsterdam A, Walker C, Westerfield M, Sive H. Combined haploid and insertional mutation screen in the zebrafish. Genesis. 2004; 40:231-240. [PubMed: 15593329]

Wienholds E, van Eeden F, Kosters M, Mudde J, Plasterk RH, Cuppen E. Efficient target-selected mutagenesis in zebrafish. Genome Res. 2003; 13:2700-2707. [PubMed: 14613981]

Wu X, Li Y, Crise B, Burgess SM. Transcription start regions in the human genome are favored targets for MLV integration. Science. 2003; 300:1749-1751. [PubMed: 12805549]

Yan YL, et al. A zebrafish sox9 gene required for cartilage morphogenesis. Development. 2002; 129:5065-5079. [PubMed: 12397114]

Yoon C, Kawakami K, Hopkins N. Zebrafish vasa homologue RNA is localized to the cleavage planes of 2- and 4-cell-stage embryos and is expressed in the primordial germ cells. Development. 1997; 124:3157-3165. [PubMed: 9272956]

Young RM, Marty S, Nakano Y, Wang H, Yamamoto D, Lin S, Allende ML. Zebrafish yolk-specific not really started (nrs) gene is a vertebrate homolog of the Drosophila spinster gene and is essential for embryogenesis. Dev Dyn. 2002; 223:298-305. [PubMed: 11836794]

Zwaal RR, Broeks A, van Meurs J, Groenen JT, Plasterk RH. Target-selected gene inactivation in Caenorhabditis elegans by using a frozen transposon insertion mutant bank. Proc Natl Acad Sci U S A. 1993; 90:7431-7435. [PubMed: 8395047] 


\section{Introduction to the new volume}

Since the initial publication of this chapter in 2004, additional methodologies have been developed which could improve and/or complement the original retroviral-mediated insertional mutagenesis. Retroviral vectors have also been shown to be useful for goals other than mutagenesis. In addition, retroviral-mediated insertional mutagenesis has been applied to zebrafish for use in reverse genetics as well as forward screening. Finally, the insertional mutant collection described herein has been screened by a number of labs to find a host of mutants (with genes already identified) with developmental and/or growth defects affecting the eye, liver, skin, craniofacial skeleton, kidney, myeloid cells, hematopoeitic stem cells, and axon pathfinding, as well as mutants with defects in the cell cycle or DNA damage response, altered ageing properties, and modulated cardiac repolarization. The major complementary approaches and new uses of this technique include:

- Pseudotyped retroviruses have been used to deliver enhancer trap vectors, allowing selection of insertions in or near genes with particular expression patterns. The mutagenicity of these vectors has yet to be determined, but they are useful purely on the basis of generating a large number of transgenic lines with visible reporters (e.g. GFP) expressed in interesting patterns and to learn about regulatory information in genomic organization.

- Gain-of-function vectors have been designed to allow for dominant genetic screens. Thus genes whose overexpression results in phenotypes of interest can be efficiently identified.

- Retroviruses can be used to make a library of insertions in which hundreds of thousands of mapped insertions can be recovered from frozen sperm samples. Such libraries could serve as on-the-shelf reverse genetic resources, whereby on can obtain a mutation in nearly any gene by simply recovering an insertion in that gene from the frozen sperm, similar to the use of gene-trap insertions and genome-wide gene targeting in ES cells in the mouse.

- Transposons have been shown to be nearly as effective transgenesis vectors as retroviruses and thus may be used in similar screens - both for mutagenicity and gene traps and enhancer traps. It is possible that retroviruses and transposons could have different insertion site biases, making them important complementary technologies for genome-wide screening. 


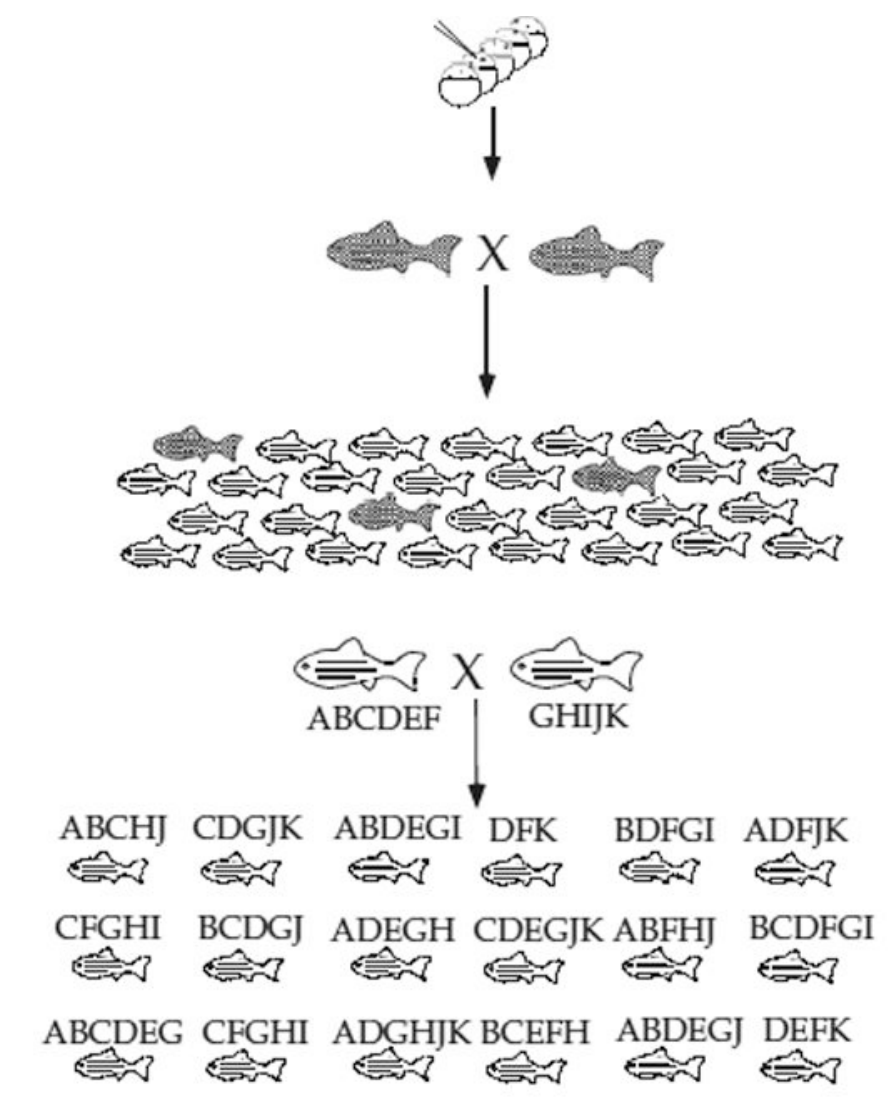

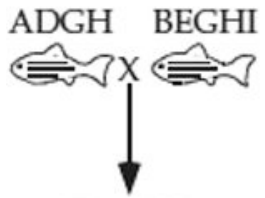

$\mathrm{G}$ and $\mathrm{H}$ homozygosed

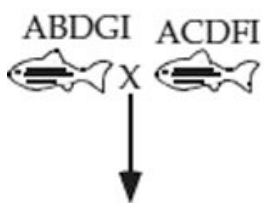

A and I homozygosed
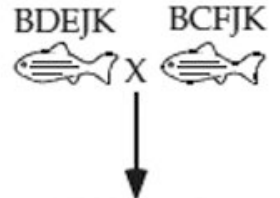

$\mathrm{B}, \mathrm{J}$ and $\mathrm{K}$

homozygosed

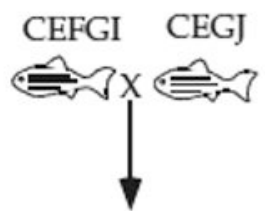

$\mathrm{C}, \mathrm{E}$ and $\mathrm{G}$ homozygosed
ADEFK DEFHJ

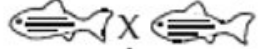

D, E and F homozygosed

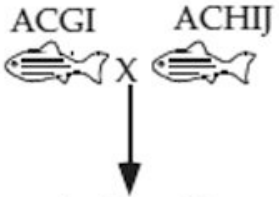

A, C and I homozygosed
1. Inject pseudotyped virus into blastula-stage embryos

2. Raise and inbreed founders

3. Raise F1 pools and isolate tail DNA from individual fish. Use quantitative pcr to identify fish with the most insertions

4. Cross multi-insert F1 fish to each other to generate $\mathrm{F} 2$ pools with10-20 insertions, in which half of the fish have any given insertion

Figure 1.

Insertional mutagenesis breeding scheme. 

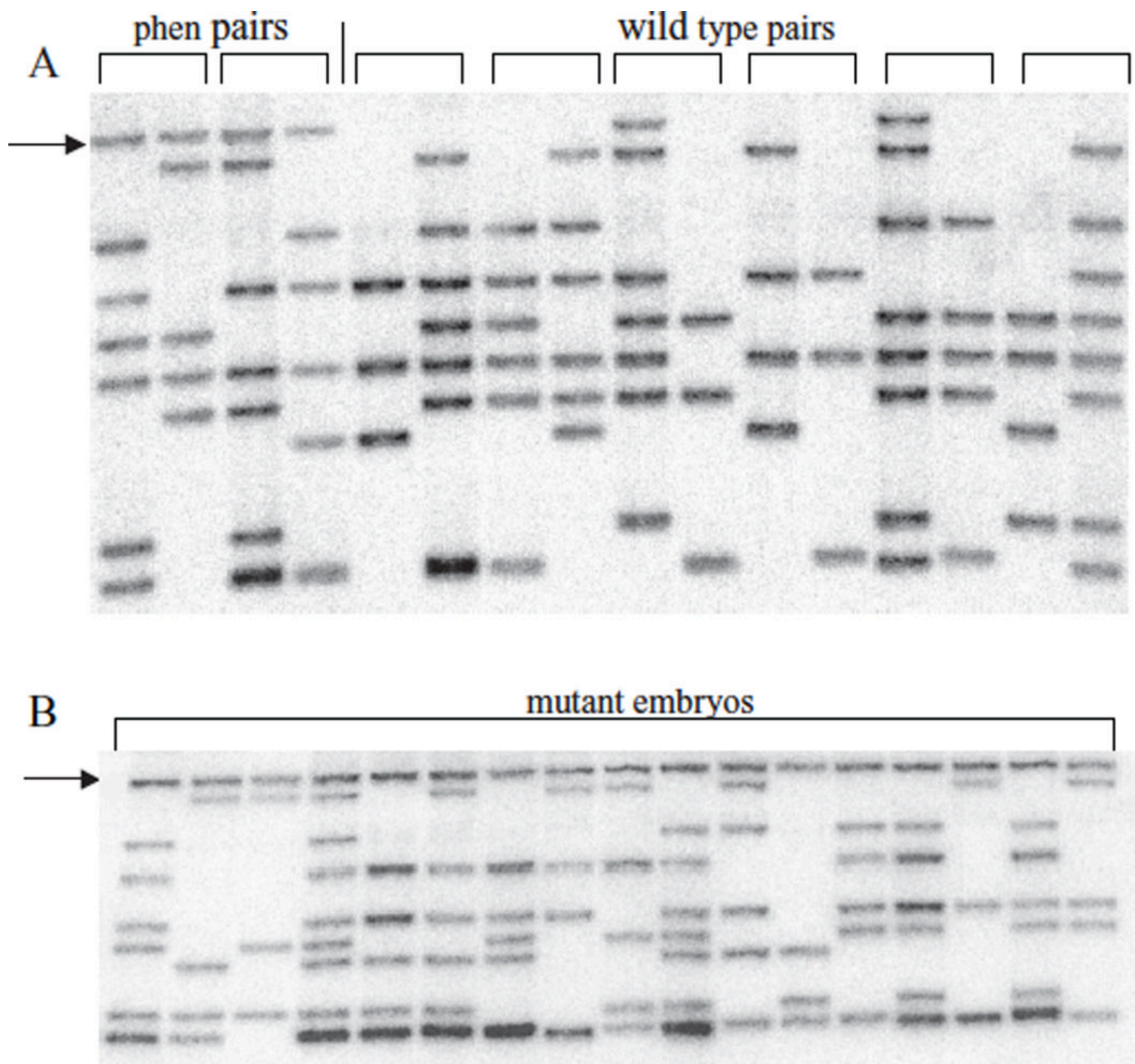

Figure 2.

Identification of the mutagenic insert. A. Southern analysis of DNA prepared from tail fins of $\mathrm{F}_{2}$ fish; the arrow indicates an insert that is homozygosed in phenotypic pairs but not any of the wild type pairs. B. Southern analysis of DNA from individual mutant embryos from the $2^{\text {nd }}$ phenotypic pair in $2 \mathrm{~A}$; the arrow indicates that the same insert is also present in all of the mutant embryos. 

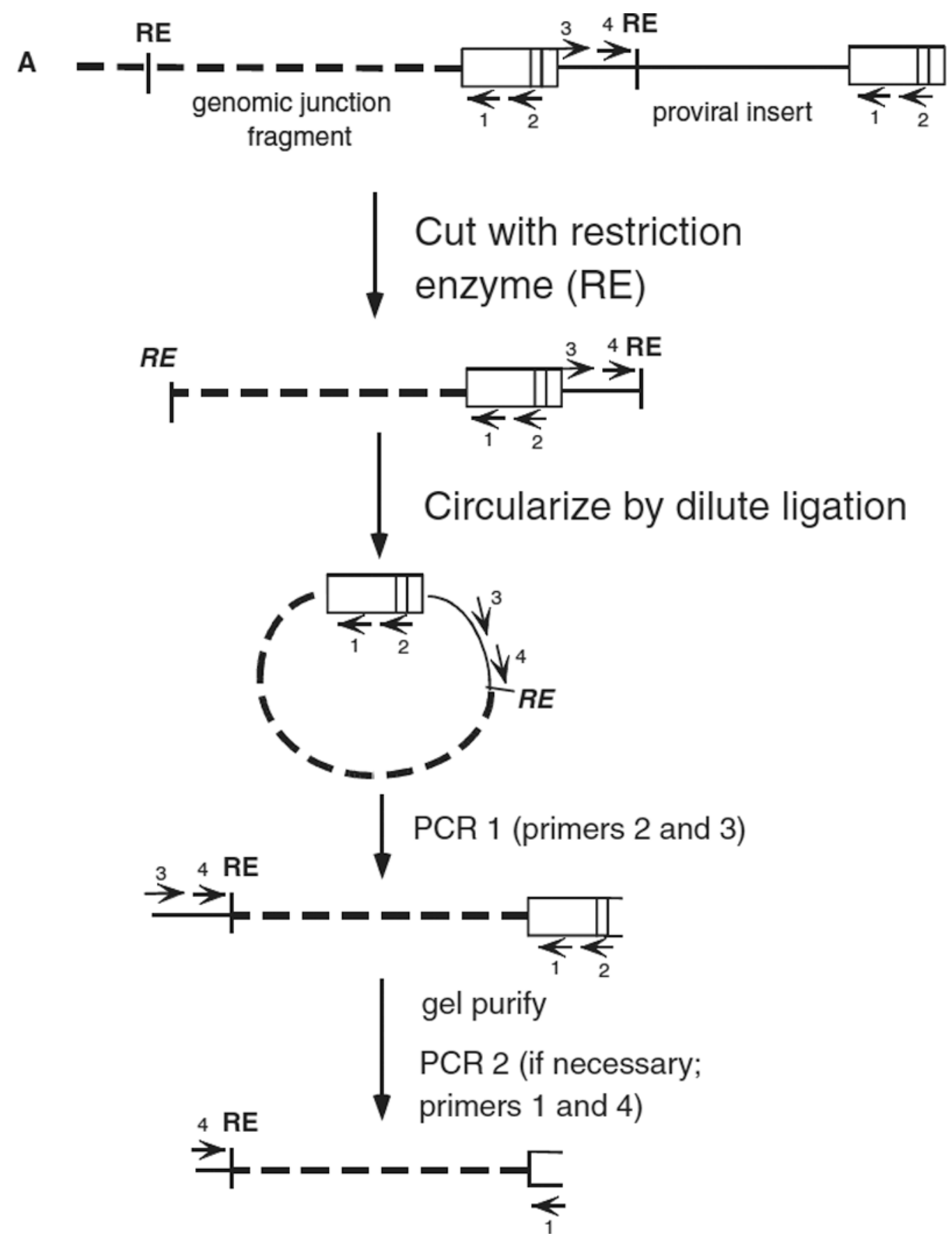
A

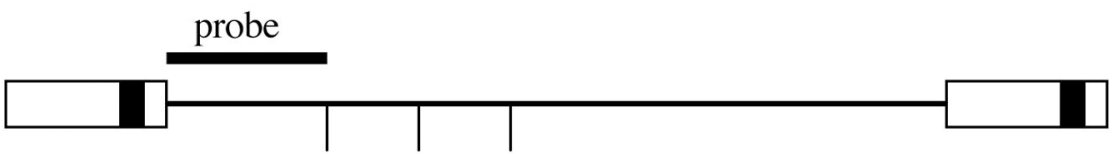

Taq I Bgl II Nco I

B

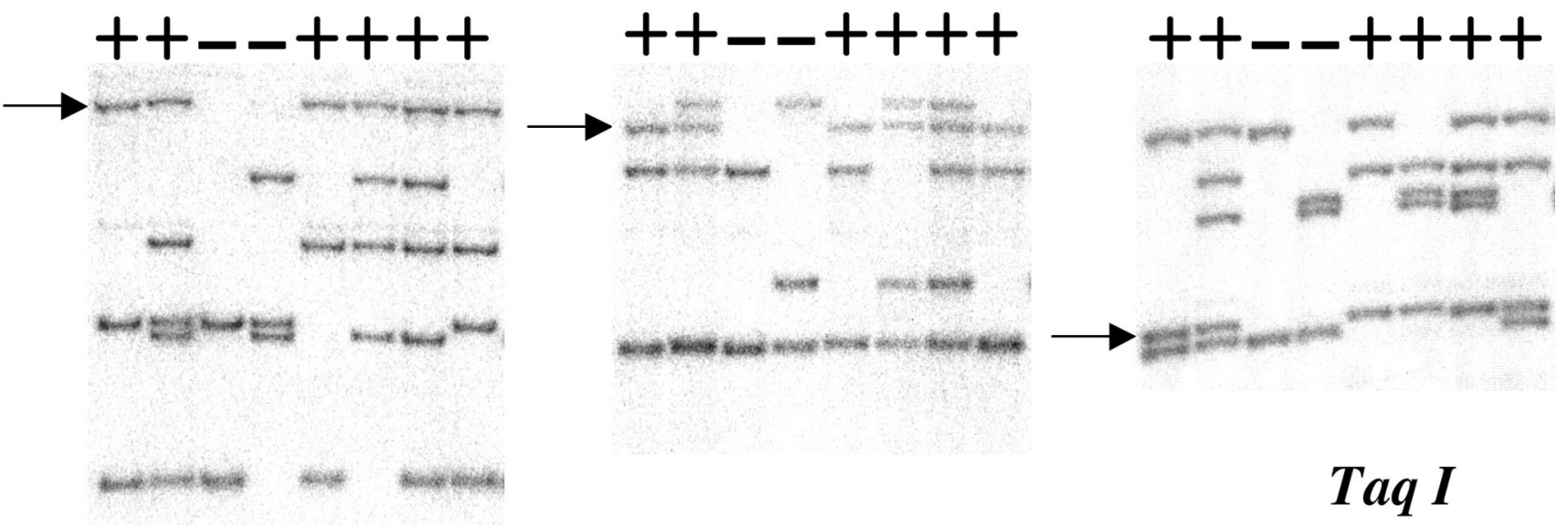

Bgl II

Nco I

Figure 3.

Inverse PCR. A. Schematic of the inverse PCR process. B. Selection of the correct enzyme to use. For an insert already identified as in figure 2, DNA from several tails already known to be positive or negative for that insert is analyzed by Southern after digestion with different restriction enzymes. In this example, while the junction fragments with $B g I I I$ and Nco I are too big to successfully amplify by inverse PCR, the Taq I junction fragment should amplify easily. 
A
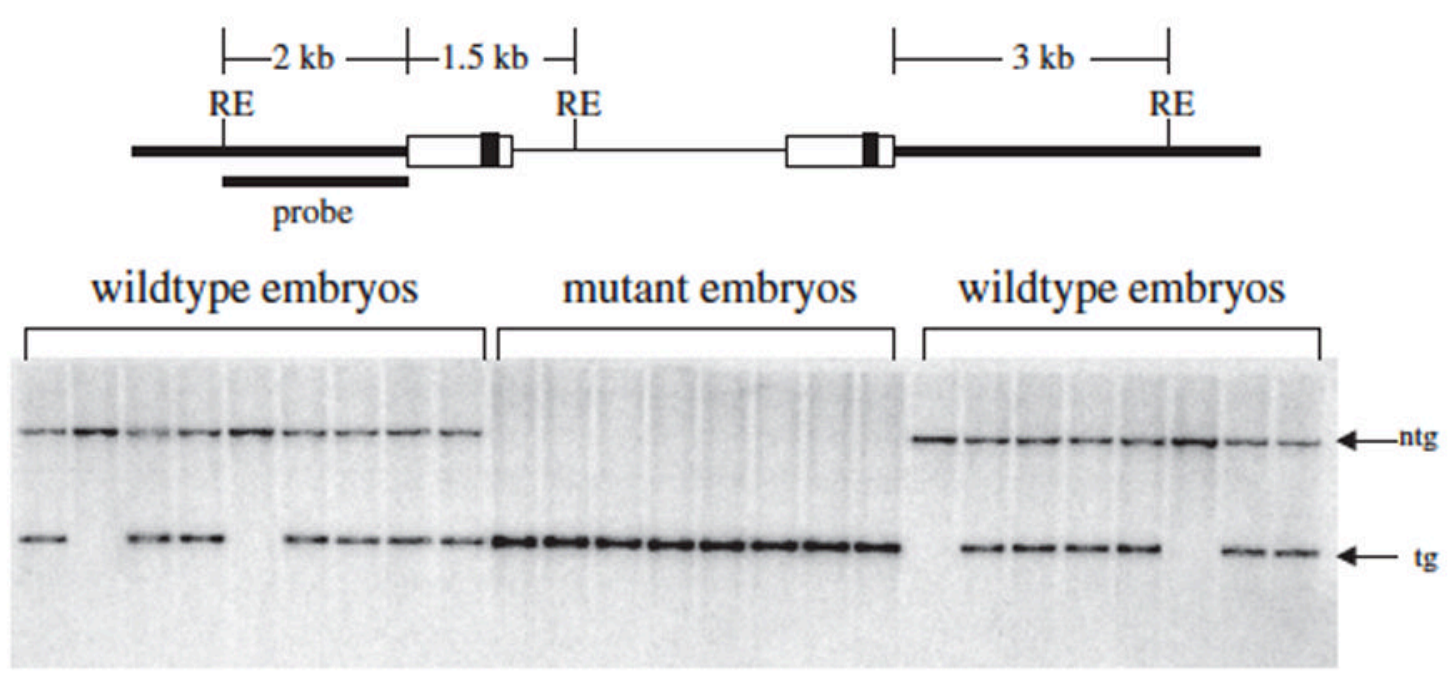

B

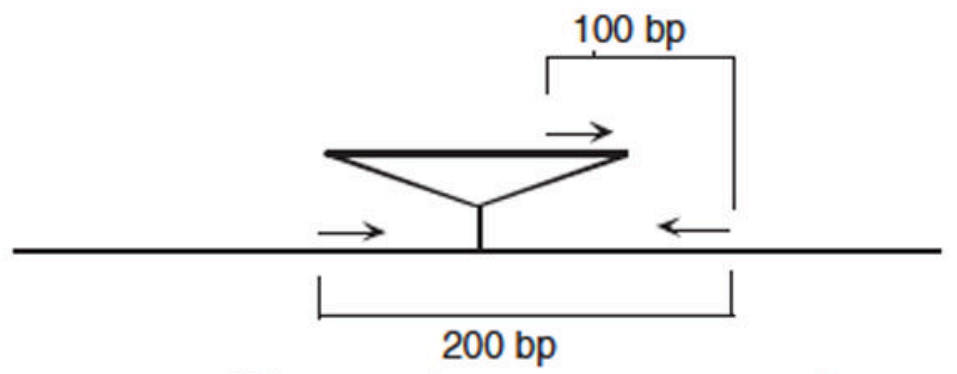

\section{mutant embryos}

wildtype embryos

mutant embryos wildtype embryos

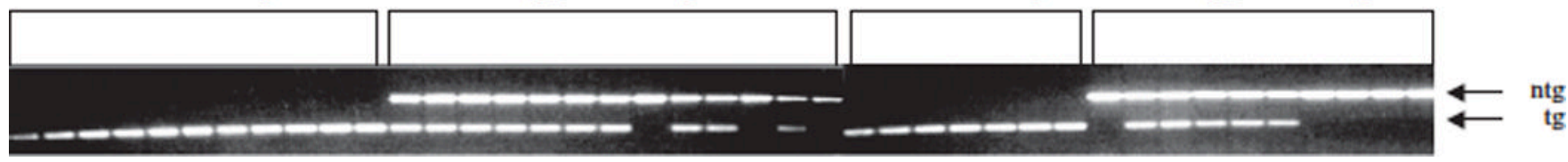

Figure 4.

Tight linkage assays. In either assay, mutant embryos should always be homozygous for the insert, while wild type embryos never should be. A. Southern analysis of DNA prepared from individual wild type or mutant embryos. The sequence of the junction fragment on one side of the virus is used as the probe. In this example, insert-bearing chromosomes (tg) will give a $3.5 \mathrm{~kb}$ band, while non-insert chromosomes ( $\mathrm{ntg}$ ) will give a $5 \mathrm{~kb}$ band. Thus each embryo can be genotyped as homozygous for the insertion (smaller band only), heterozygous (both bands), or homozygous non-insertion (larger band only). B. PCR analysis of DNA prepared from individual wild type or mutant embryos. The PCR reaction is run with 3 primers, such that (as with the Southern method) the presence of either chromosome is indicated by a unique sized band. 


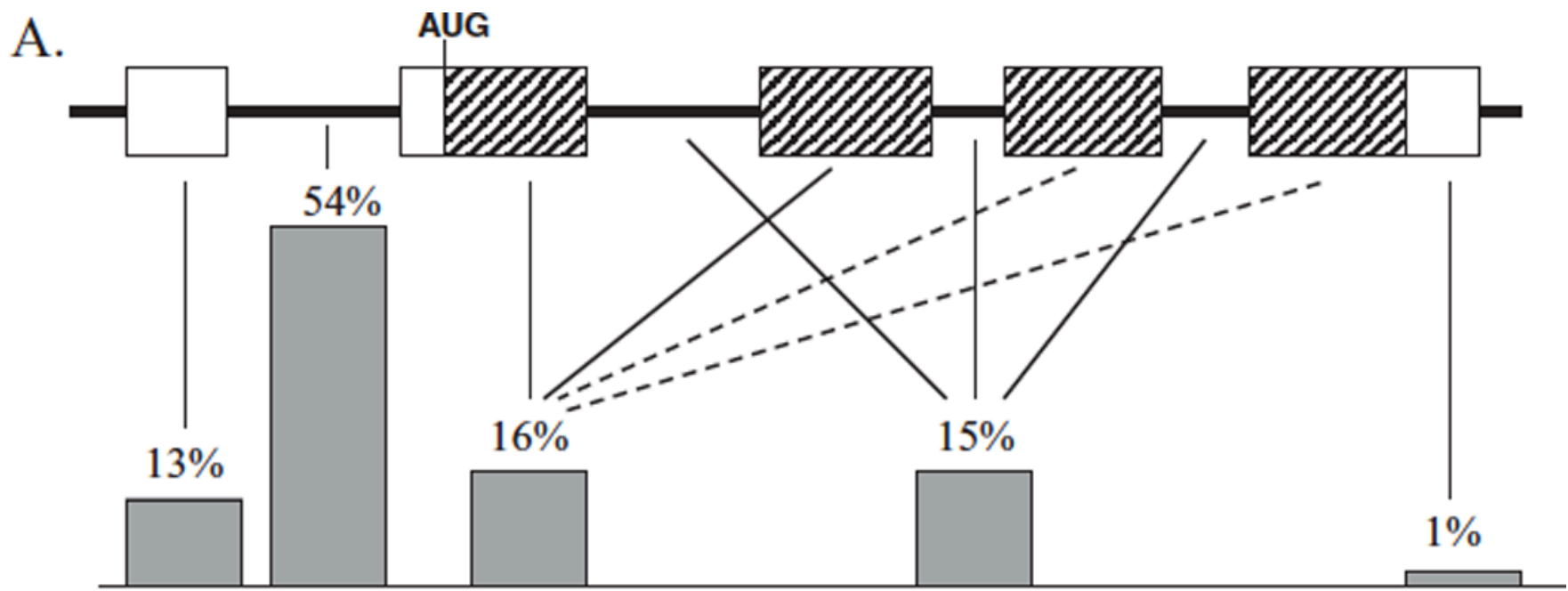

B.

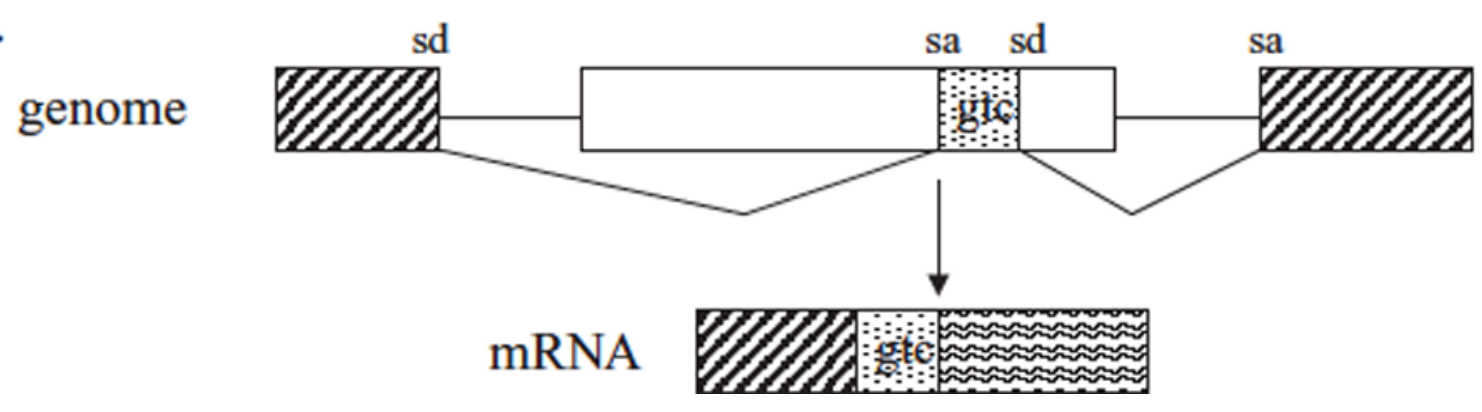

Figure 5.

Mutagenic insertion sites. A: Distribution of mutagenic insertions from 413 insertional mutants in 298 different genes. White boxes indicate 5' and 3' untranslated regions (UTR), striped boxes indicate coding exons, lines between boxes indicate introns. While the sample gene here has an untranslated first intron (the first white box), in about half the cases the first exon contains the initiation codon. The percentage of mutagenic insertions which lie in the 5' UTR or promoter, the first intron, coding exons, downstream introns, or the 3' UTR, is shown below the gene. B: Consequence of gene trap event. If the insertion is in an intron in the correct orientation, the splice donor from the previous exon can splice to the gene trap cassette, and then splice out to the next endogenous exon, thus creating a frameshift. Striped boxes, exons; large white box, provirus; stipled box (gtc), gene trap cassette; sd, splice donor; sa, splice acceptor; wavy-lined box, exon with frameshift mutation. 
GT186 viral packaging cells

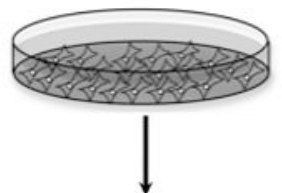

High-titer pseudotyped MLV

Founder (mosaic)
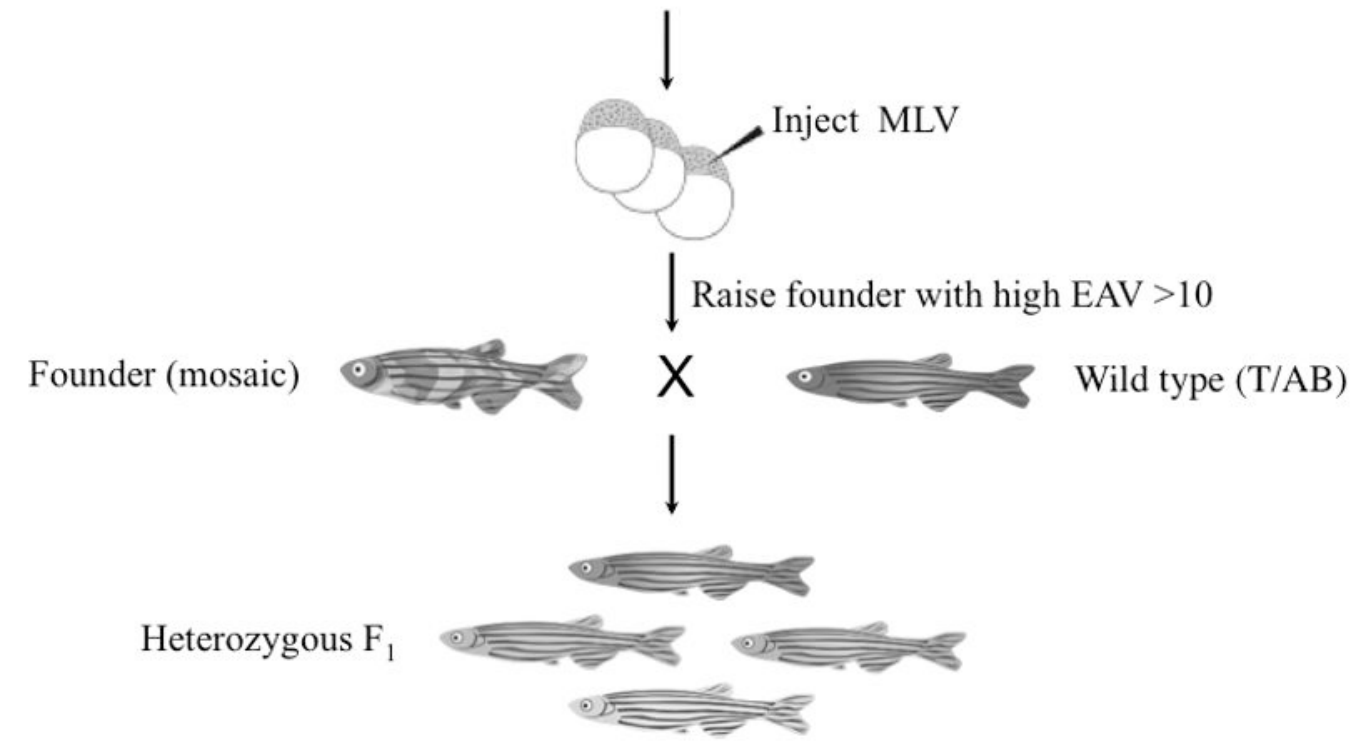

Mutant library (frozen sperm)

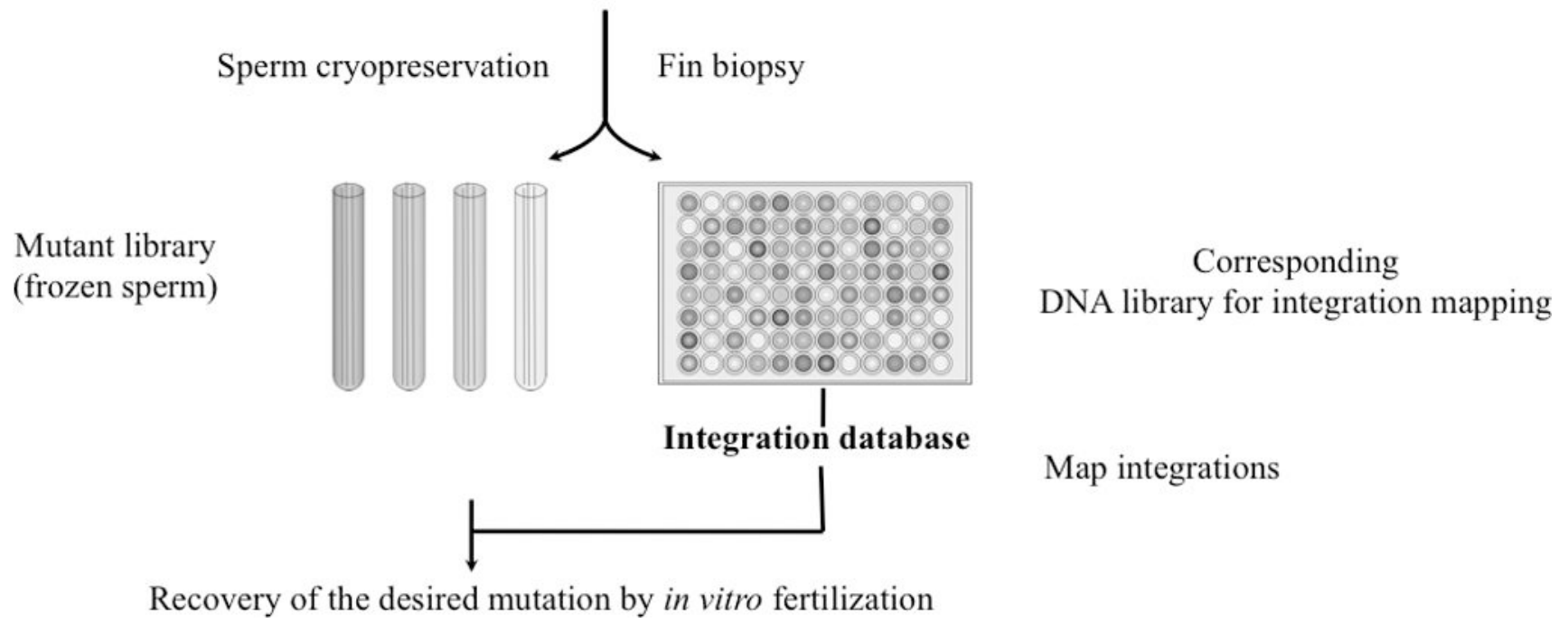

Figure 6.

Retroviral mapping pipeline. Blastula stage embryos are injected with the pseudotyped MoMLV. Infection rate is determined by quantitative PCR (qPCR) and founder fish with high infection rate are raised. Founders are crossed to wild-type fish and male $F_{1}$ fish are raised. Sperm samples are cryopreserved from the $F_{1}$ fish, integrations are mapped in the corresponding DNA library, integrations are assigned to the corresponding sperm samples. Desired mutations are recovered by in vitro fertilization of the corresponding frozen sperm. 

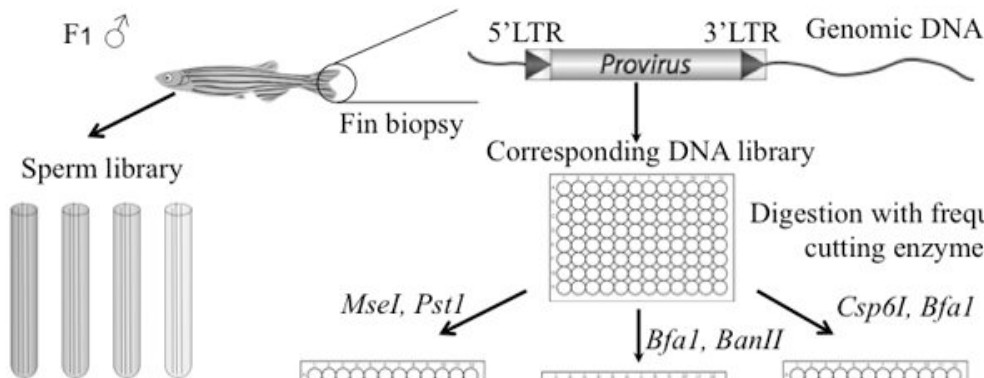

Assign the integrations to the respective sperm samples using barcodes
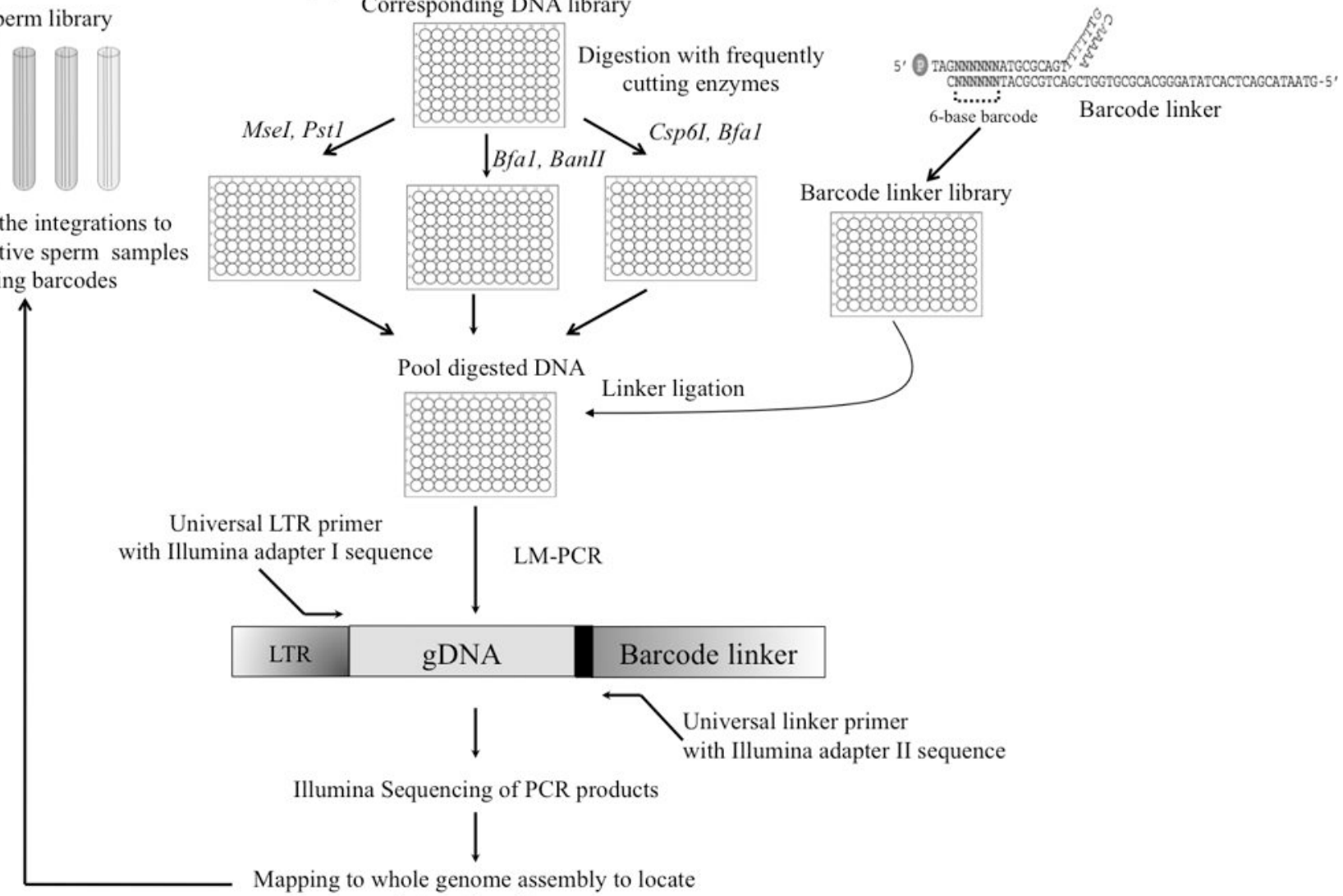

Universal LTR primer

LM-PCR

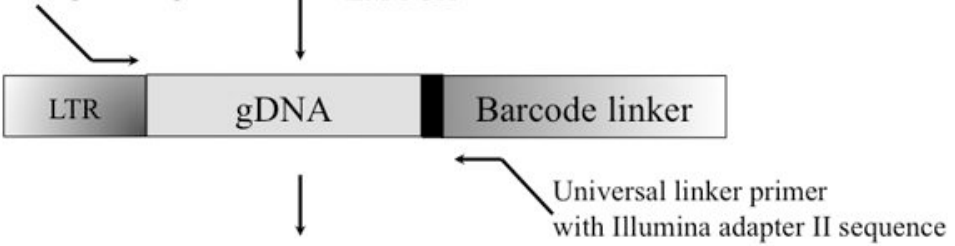

Illumina Sequencing of PCR products

Mapping to whole genome assembly to locate

Integration sites

Figure 7.

High-throughput strategy to identify retroviral integrations using next-generation sequencing. DNA samples corresponding to individual sperm are samples are separately digested with three sets of restriction enzymes to fragment genomic DNA. The digested samples are then ligated with a DNA linker containing a unique 6-bp barcode to index each $\mathrm{F}_{1}$ fish. The linker-ligated DNA samples are amplified by LM-PCR using LTR and linker specific primers to amplify the adjacent genomic DNA sequence. The 5' ends of the LTR and linker primers also contain adapter sequences required to bind to the Illumina flow-cell for sequencing. Illumina sequencing can generate up to $150 \mathrm{bp}$ of mappable sequences using the paired-end method. These paired-end sequences are aligned to the latest assembly of the zebrafish genome and the integrations are assigned to corresponding sperm samples using the index of unique barcodes. 